\title{
Pain acts through fatigue to affect participation in individuals with multiple sclerosis
}

\author{
Shahnaz Shahrbanian $^{1,2} \cdot$ Pierre Duquette $^{3}$ - Sara Ahmed ${ }^{1,4}$ - Nancy E. Mayo ${ }^{1,4}$
}

Accepted: 3 August 2015/Published online: 9 August 2015

(C) Springer International Publishing Switzerland 2015

\begin{abstract}
Purpose The symptoms of multiple sclerosis (MS) often cause physical and mental dysfunction, which interferes with a person's ability to participate in life's roles. Identification of the strength of the contributors to participation would help prioritize intervention approaches for its improvement. The objective of this study was to estimate the extent to which pain and other MS-related symptoms, physical function, psychological variables, and individual characteristics predict participation in people with MS.

Methods This was a cross-sectional study. Study sample was a center-stratified random sample of 188 persons with MS. Subjects completed a battery of self-report and performance-based measures that assessed participation and domains affecting participation. To model the relationships among variables, a conceptual framework based on the Wilson and Cleary model was tested. Structural equation modeling aimed at identification of the predictors of participation within the hypothesized theoretical model.

Results Fatigue $(\beta=0.63, p<0.0001)$, physical function $(\beta=0.37, p<0.0001)$, and psychological variables

Shahnaz Shahrbanian

shahnaz.shahrbanian@mail.mcgill.ca

1 Faculty of Medicine, School of Physical and Occupational Therapy, McGill University Health Center, McGill University, Royal Victoria Hospital Site, Ross Pavilion R4.27, 687 Pine Ave W, Montreal, QC H3A 1A1, Canada

2 Faculty of Physical Education and Sport Science, Buali Sina University, Hamedan, Iran

3 Notre-Dame Hospital (CHUM), University of Montreal, Montreal, Canada

4 Division of Clinical Epidemiology, McGill University Health Center, Royal Victoria Hospital, Montreal, Canada
\end{abstract}

( $\beta=0.15, p=0.04$ ) were found to be as significant direct predictors of participation. Pain $(\beta=0.4, p<0.0001)$ and age $(\beta=0.12, p<0.0001)$ were significant indirect predictors through fatigue and physical function, respectively. Together these effects explained $88 \%$ of the variance of participation, $35 \%$ of the variance in psychological variables, and $29 \%$ of the variance in physical function.

Conclusion Fatigue, physical function, pain, and psychological variables were most important contributors for participation in persons with MS.

Keywords Multiple sclerosis - Pain - Participation · Fatigue · Wilson-Cleary model · Structural equation modeling $\cdot$ Rehabilitation

\section{Introduction}

Multiple sclerosis (MS) is the most common disabling neurological disease among young adults in North America [1-3]. MS affects nearly all aspects of life [1-3]. Symptoms of fatigue, pain, and depression interfere with capacity for physical effort, psychological functioning, work, leisure activities, and lifestyle, making MS one of the most intrusive illnesses [4]. Increasingly, the ultimate target of rehabilitation interventions is on enhancing participation in life's roles [5], an outcome known to be contributing strongly to quality of life (QOL) [6, 7].

The World Health Organization's (WHO) International Classification of Functioning, Disability and Health (ICF) defines participation as "involvement in life situations" [8]. Participation indeed represents the societal perspective of functioning as it covers a number of aspects that are more likely to be performed with others [8]. Participation is considered as a global construct that covers personal and 
household management, leisure activities, community involvement, work, and relationships with others [9]. The concept of participation as a multidimensional health outcome has been studied extensively in other chronic conditions $[6,7,10,11]$, but less so for people with MS [12, 13]. Existing literature on MS suggests that people with MS have less involvement in social activities, lower perceptions of social support, less marital satisfaction, and a shrinking social network [1, 3, 13]. Knowledge of the contributors to participation and how they interact can help focus rehabilitation interventions so that, ultimately, QOL can be optimized.

Participation is influenced by impairments and activity limitations [8]. Although fatigue is the most reported and disabling symptom of MS, pain is also a common symptom reported by almost $50 \%$ of people with MS at some point of their disease course [1-3]. The results of a meta-analysis have indicated that individuals with MS who experience pain are significantly more likely to report a decreased employment rate than MS people who are pain free [14]. Considering that in comparison with people with MS who do not have pain, people with pain report more psychological distress and poorer physical and mental function $[15,16]$, and it is assumed that pain may catalyze the social isolation of persons with MS [15-18].

Like participation, pain is conceptualized as a multidimensional phenomenon [19], necessitating the developing an interactive multidimensional framework that simultaneously evaluates the complex interrelationships among all contributing factors [19]. There are several theoretical frameworks that have been developed to explain the relationship among health outcomes. One of the commonly applied conceptual models is the Wilson-Cleary model (WCM) [20]. WCM is an interactive multidimensional theoretical model of health-related quality of life (HRQL) that proposes consequences of health conditions. WCM explains the linear interrelationship between and among biological and physiological variables, symptoms status, functional status, general health perception, and overall QOL [20]. The model also indicates that each of these components can be affected by individual and environment characteristics [20].

A broad-based examination of the key determinants of participation in people with MS has not been widely studied, nor has the relative contribution of various clinical, personal, and environmental determinants within an interactive theoretical framework that considers all contributing factors in this population been explored. Previous studies have considered only a relatively limited range of potential variables that might account for the variability in participation [15-18]. In addition, previous works have focused only on a single dimension of contributing factors [15-18]. More effort needs to be made to conceptualize all the dimensions of participation for women and men with MS.
The objective of this study, therefore, was to contribute evidence to support a biopsychosocial conceptual framework of participation in the context of MS by clarifying the direct and indirect effects of pain and other MS-related symptoms, physical function factors, psychological variables, environmental factors, and individual characteristics on participation. It was hypothesized that MS-related symptoms, specifically fatigue and pain and physical functioning would be the most important identified predictors. A preliminary conceptual model to assist in the definition and measure of variables that are important in order to create a priori hypotheses about the interactions among different factors and role participation is illustrated in Fig. 1.

\section{Methods}

\section{Participants}

The target population for this study consisted of persons with a diagnosis of MS since 1995. The available population was all men and women registered at the three major MS clinics in Greater Montreal including Montreal Neurological Hospital (MNH), Centre Hospitalier de l'Université de Montréal (CHUM), and Clinique Neuro Rive-Sud (CNRS). The time frame was stratified into three eras: 1995-1999, 2000-2004, and 2005-2006 to ensure that people from each era were included. The number of people diagnosed with MS since 1995 at the three clinics was 1950 . A center-stratified random sample of 550 individuals with MS was drawn, of which 364 were contacted. From those who were contacted, the first 192 who responded were only included due to the limited budget. Following exclusion of three people with incomplete data and one person who did not attend the evaluation session, 139 women and 49 men comprised the study sample (52\% of those contacted). No significant difference was found between responders $(n=188)$ and nonresponders $(n=176)$ on age, sex, MS severity, date of diagnosis, and duration of symptoms. To reflect or resemble the underlying epidemiology of MS [3], the sample consisted of substantially more women than men (the ratio 2.8:1). Participants who had a relapse in the preceding month, participants younger than 18 years old, people with severe cognitive impairments, and those with preexisting health conditions affecting functioning were excluded from participating in the study.

\section{Procedure}

Eligible people were sent a letter of invitation from the director of each related MS clinic. A research coordinator later contacted the candidates to verify whether they met 
Fig. 1 Initial hypothesized model

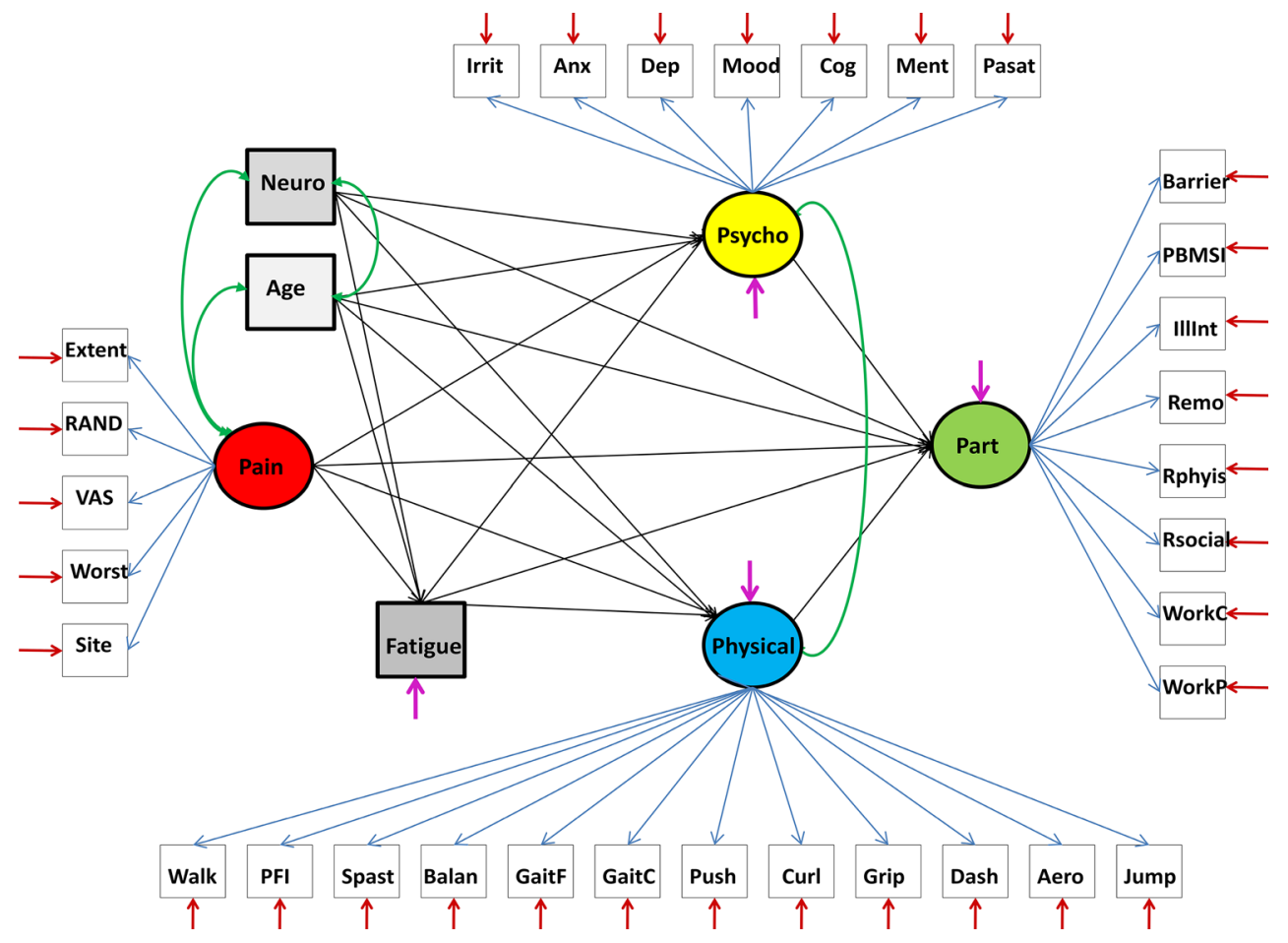

the eligibility criteria, and invited them to participate if they did. If persons consented to participate, an appointment was arranged for assessment of study measures. If they refused to participate, socio-demographic and clinical information were collected. On the day of testing, participants were asked to complete several patient-reported outcome measures and performance-based measures representing the domains identified in the WCM. Study protocol, measures, and procedures were approved by the ethics committee of each participating hospital, and informed consent was obtained.

\section{Measurement}

Measures of study are listed in Table 7.

\section{Characteristics of the individuals}

The personal characteristics of gender, age, education, and employment were recorded on the day of testing using the socio-demographic questionnaire.

\section{Biological and physiological variables}

The clinical records of each person were reviewed to obtain data on MS type, years since MS diagnosis, and symptoms onset. Persons also were asked to report whether they used disease-modifying therapy (DMT). The severity of disease was assessed using the Expanded Disability Status Scale
(EDSS), which has become the standard measure for classification of MS-related disability, ranging from 0 (no disability) to 10 (maximum disability) [21]. EDSS was administered by an independent neurologist who was not a part of the study.

A nine-item measure to indicate neurological impairments was created using Rasch analysis reflecting lesion location, and clinical information such as EDSS, MS type, the use of DMT, and disease duration [22].

\section{Symptoms status}

Pain Several measures of pain were used to create a latent variable for pain component. 0-10 Numeric Rating Scale (NRS) was used to assess pain intensity in the past week [23]. Pain location and distribution was quantified using the Margolis Drawing Rating System (MRS) [24]. 0-10 Visual Analogue Scale (VAS) was used to assess muscle pain. Bodily pain (BP) was measured using the BP subscale from the RAND 36-Item Short Form Health Survey (RAND-36) [25, 26].

Psychological variables Anxiety, depressive symptoms, irritability, mood, memory, concentration, processing speed, sustained attention, and mental health made up a latent variable for psychological variables. The Hospital Depression and Anxiety Scale (HADS) was used to assess depression and anxiety in the sample [27]. Irritability was measured using a specific irritability index created from 
Rasch analysis of Psychiatric Symptom Index (PSI) [28]. The scale comprised four items ranging from 1 "never" to 4 "very often." A maximum total score of 16 represents the most irritability symptom. VAS and the Perceived Deficits Questionnaire (PDQ) were used to assess mood and cognitive symptoms, respectively [29]. The Paced Auditory Serial Addition Task (PASAT) was used to assess innate processing speed and sustained attention in the sample [30]. The five-item Mental Health Index (MHI-5) subscale (also called "nonspecific psychological distress") from the RAND-36 was used to assess mental health in the sample $[25,26]$.

Fatigue Several measures were used to create a latent variable of fatigue using Rasch analysis. They included vitality subscale (VIT) of the RAND-36 [25, 26], the thinking/fatigue subscale of the Functional Assessment of Multiple Sclerosis (FAMS) [31], the Modified Fatigue Impact Scale (MFIS) from the Multiple Sclerosis Quality of Life Inventory (MSQLI) [32], and the Multidimensional Fatigue Inventory (MFI) [33]. The final fatigue measure included 14 items with a total score ranging from 0 to 43 and the $90 \%$ reliability estimate.

\section{Functional status}

Participation We created a latent component of participation using several corresponding representative measures of work, lifestyle disruptions, exercise, and leisure activities. Role physical (RP), role emotional (RE), and social functioning (SF) subscales from the RAND-36 were used to identify problems with work or other regular daily activities in the sample [24]. Information was also available on work time (hours of paid worked) and work activity (hours taking care of people and worked). The Exercise Barriers Scale (EBS) was used to assess exercise barriers and facilitators in the sample [34]. The Preference-Based Multiple Sclerosis Index (PBMSI)-modified from the Preference-Based Stroke Index (PBSI) [35]—was used to assess participants' ability to accomplish activities such as recreational activities and driving considering their own health on the day of evaluation [22]. Finally, Illness Intrusiveness Rating Scale (IIRS) measured the illness intrusiveness [36], which has been defined as "illness and treatment-induced lifestyle disruptions that interfere with continued involvements in valued activities and interests" [36].

Physical function A latent variable for physical function was created from walking capacity, gait speed (comfortable and fast), balance, spasticity, exercise capacity, lower extremity power, grip strength, physical function, upper limb and abdominal muscle endurance, and upper extremity dysfunction. The six-minute walk test (6MWT) [37] and gait speed test [38] were used to assess walking capacity and gait speed (comfortable and fast) in the sample, respectively. EQUI Scale [39] was used to measure balance, and the Modified Ashworth Scale (MAS) [40] was used to assess spasticity in the sample. The Modified Canadian Aerobic Fitness Test (MCAFT) [41] was used to assess exercise capacity in the sample. Lower extremity power, grip strength, and physical function were assesses by the vertical jump test [42], the Jamar TM dynamometer (JTMD) [43], and the Physical Functioning Index (PFI) of the RAND-36 [25, 26], respectively. Push-up and partial curl-up tests were used to assess the endurance of upper limb and abdominal muscles [41]. The Disabilities of the Arm, Shoulder and Hand (DASH) Scale was applied to measure upper extremity dysfunction [44].

\section{Statistical analyses}

Descriptive statistics were used to describe the sample and summarize data. The potential for selection bias was tested using Chi-square test for categorical variables, $t$ test for continuous variables with homogenous variances, and $\mathrm{U}$ Mann-Whitney test for continuous variables with non-homogenous variances. Associations between all variables were assessed using Spearman and Pearson correlation coefficients for categorical and continuous variables, respectively.

To test the Wilson-Cleary theoretical conceptual framework against the observed data, structural equation modeling (SEM) was considered the most appropriate option of analysis. SEM, a combination of factor analysis, path analysis and regression, is a powerful and advanced statistical approach that has the ability to simultaneously evaluate a priori hypotheses concerning both direct and indirect relationships between and among constructs and variables within multiple alternative models [45].

The assumptions of SEM, such as collinearity and multivariate normality, were tested prior to the analysis. Collinearity occurs when two or more indicators in the model are highly correlated. Collinearity provides redundant information about the response and so increases the standard errors of parameter estimates and decreases reliability [45]. Correlation matrices between all pairs of indicators were constructed to assess for collinearity. If correlation coefficients between two indicator variables were close or larger than 0.8 , one of them was removed from the model [45]. Regarding multivariate normality, since many construct-specific measures were not normally distributed, all models were estimated using robust maximum likelihood (MLR), which produces more accurate standard error estimates with non-normally distributed variables and a more accurate Chi-square statistic [45]. 
The goodness of fit of the model is examined using several fit indices including the Satorra-Bentler scaled Chisquare statistic, Comparative Fit Index (CFI), TuckerLewis Fit Index (TLI), Root Mean Square Error of Approximation (RMSEA), and the Standardized Root Mean Squared Residual (SRMR) [46]. A $p$ value smaller than 0.05 for Chi-square test, a cutoff value 0.95 or larger for CFI and TLI; a p value smaller than 0.06 with a cutoff value 0.90 for RMSEA; and a $p$ value below 0.08 for SRMR suggest an adequate fitting model [46].

Evaluation of the alternative models was based on the theoretical knowledge, along with the statistical criteria for adding or removing paths. We used Lagrange Multiplier test for adding the paths. A critical ratio ( $t$ value) that exceeds +1.96 or fall below -1.96 along with a nonsignificant $p$ value as well as the Wald test was used for eliminating the paths [45].

A major restriction of SEM is its requirement for a large sample size that is somewhat dependent on model complexity [45, 46]. In order to estimate parameters with reliability, rules of thumb suggest 200 cases or 10 subjects per parameter being estimated [45]. As the number of available variables was large with respect to the available sample size, a set of steps were used to drop some of them. First, the correlation matrix was examined to identify variables with weak association with the outcome. Variables that did not correlate to the main outcome were eliminated. In addition, some of the variables were removed from the model due to collinearity. Of the correlated variables with strong correlation to the outcome, those that severely violated the assumption of normality were eliminated as well.

The power of the model was computed using the MacCallum method, which is an extension of Satorra and Saris' power analysis procedure [47].

Statistical analyses were conducted using the Mplus statistical software version 7.1 [48] for SEM model and fit indices, and Statistical Analysis Systems (SAS) version 9.2 [49] for descriptive statistics and correlation matrix. Mplus has the advantage of handling missing data as well as data that are neither continues nor normally distributed [48].

\section{Results}

\section{Description of the sample}

Characteristics of the participants are presented in Tables 1 and 2, respectively.

The sample consisted of substantially more women than men (the ratio $3: 1$ ). Both men and women had mild disability with a median EDSS score of $<3$. The results of the correlation analyses showed that most variables were correlated with participation indicators. Fatigue showed high correlation with physical function, and participation indicators (Table 3).

\section{Structural equation modeling}

\section{Construction of the initial hypothesized path model}

A total of 32 variables representing the different domains of the WCM were initially included in the analysis. The hypothesized measurement model consisted of four latent variables, physical function, psychological variables, pain, and participation. Indicators for each latent variable are presented in Table 2 and Fig. 1. A number of variables, such as fatigue and neurological impairments, were further included as single-indicator latent variable to the structural part of the model. The latent variables of fatigue and neurological impairments, created with single indicators, were assigned reliabilities of 90 and $74 \%$, respectively [22].

The strict interpretation of the WCM is a progression from biological factors to symptoms to functioning. Thus, the model was built in phases beginning with the paths from the neurological impairment, to the fatigue, to the physical function, and finally to the outcome, participation. Gender and other personal factors such as education or living situation were not included in the analysis as there was no significant difference between these variables and participation indicators in this study. The original hypothesized structural model is illustrated in Fig. 1.

\section{Simplified path model}

Table 4 sets out the model progression. As expected, due to the complexity of the model, the fit of the original hypothesized path model was not adequate. Thus, several steps were taken for improving the model fit. The RAND-PFI, DASH, gait speed (fast) were removed from the physical function latent, as they were highly correlated with the several other indicators of function such as walking capacity, gait speedcomfortable, and balance (Table 3). Results of factor analysis further demonstrated that not all of the indicators fit with their hypothesized latent factors. Thus, variables such as pain location, PASAT, physical capacity tests, PBMSI, the RAND-SF, the RAND-RE, and work time, and work activity were dropped from the further analysis. However, even with the exclusion of work variables from the model, information related to the work variables could still be captured using the illness intrusiveness questionnaire as a work item was already present in the illness intrusiveness questionnaire, determining the ratings of the degree to which one's illness interfere with life domains such as work and employment [36]. 
Table 1 Description of the sample on the Wilson-Cleary rubrics of personal, environmental, and biological and physiological factors $(N=188)$

\begin{tabular}{|c|c|c|}
\hline Variables & $\begin{array}{l}\text { Frequency } \\
\text { or (mean) }\end{array}$ & $\begin{array}{l}\text { Percent } \\
\text { or }[S D \text { ] }\end{array}$ \\
\hline \multicolumn{3}{|l|}{ Characteristics of the individual } \\
\hline Current age & $(42.9)$ & {$[10.2]$} \\
\hline \multicolumn{3}{|l|}{ Gender } \\
\hline Women & 139 & 74 \\
\hline Men & 49 & 26 \\
\hline \multicolumn{3}{|l|}{ Level of education } \\
\hline University & 83 & 47.4 \\
\hline High school & 54 & 30.9 \\
\hline Less than high school & 38 & 21.7 \\
\hline \multicolumn{3}{|l|}{ Living situation } \\
\hline Alone & 34 & 19.3 \\
\hline Spouse/partner & 108 & 61.4 \\
\hline Family member(s) & 32 & 18.2 \\
\hline Friend(s) & 1 & 0.6 \\
\hline Other & 1 & 0.6 \\
\hline \multicolumn{3}{|l|}{ Environmental characteristics } \\
\hline \multicolumn{3}{|l|}{ Employed } \\
\hline Yes & 122 & 70 \\
\hline \multicolumn{3}{|c|}{ Biological and physiological variables } \\
\hline \multicolumn{3}{|l|}{ MS type } \\
\hline Relapsing-remitting & 97 & 78 \\
\hline Secondary progressive & 7 & 5 \\
\hline Primary progressive & 8 & 7 \\
\hline Primary relapsing & 3 & 3 \\
\hline Clinically isolated syndrome & 9 & 7 \\
\hline Years since diagnosis & (3) & [4] \\
\hline Years since symptom onset & (9) & {$[5]$} \\
\hline MS severity (EDSS: median) & 2.4 & 2 \\
\hline \multicolumn{3}{|l|}{ Disease-modifying therapy } \\
\hline Yes & 110 & 85 \\
\hline
\end{tabular}

\section{Re-specified path model}

The model was then further revised by eliminating the direct paths from pain and age to participation, as well as pain to physical function and psychological variables. Additionally, the component of neurological impairments was removed from the model due to the nonsignificant effects of it on several paths of the model, and the mild disability level of the current sample.

\section{The final path model}

The final model is shown in Fig. 2. Overall, the results of fit indices indicated good fit of the model (Table 4). According to the model, participation was directly predicted by fatigue $(\beta=0.63, p<0.0001)$, physical function $(\beta=0.37, p<0.0001)$, and psychological variables $(\beta=0.15, p=0.04)$ (Table 5). Pain was a significant indirect predictor of participation through fatigue $(\beta=0.4, p<0.0001)$. The effect of age on participation mediated through physical function $(\beta=0.12$, $p<0.0001)$ as well. Fatigue also showed an indirect effect on participation through physical function $(\beta=0.14$, $p<0.0001)$. Together these effects explained $88 \%$ of the variance of participation, $35 \%$ of the variance in psychological variables, and $29 \%$ of the variance in physical function. Table 6 presents the maximum likelihood estimates of final model on participation. These standardized path coefficients represent the strength of the relationship among variables. The statistical power of the final model was estimated to be as high as $93 \%(N=188, d f=94$, $\alpha=0.05$, RMSEA range $=0.05-0.08$ ).

\section{Discussion}

This study modeled participation using the framework of the WCM and SEM, in a sample of people with MS. Overall, our results provided support for fatigue, physical function, and psychological variables as most important direct contributors for participation in persons with MS. Pain and age showed indirect effects.

The results of this study highlighted the importance of doing more investigations on fatigue due to its overall effect on participation so HRQL. Fatigue was found to not only contribute to the construct of participation, as defined in this study, but was also a contributor to all other variables in the model, thus confirming its role as the most disabling symptom of MS. Similar to the study by Kempen [50], our results also indicated that fatigue acts as independent determinants of physical functioning. Pharmacological and non-pharmacological interventions are currently underway to try and relieve MS fatigue [51]. Clinical practice guidelines suggest pharmacological treatments, exercise training and energy conservation techniques for managing fatigue [52]. A recently published meta-analysis compared the effectiveness of three types of interventions including exercise, education, and medication on fatigue management. Results of this study indicated that non-pharmacological interventions, i.e., exercise interventions, appeared to have stronger effects on reducing the severity of patient-reported fatigue compared with pharmacological interventions [53]. A recently published scoping review further identified a variety of exercise and behavior change interventions for MS fatigue management. Results of this review showed that both exercise and behavior change interventions demonstrate some degree of effectiveness for managing MS fatigue [54]. A cognitive- 
Table 2 Description of the sample on the Wilson-Cleary rubrics of symptoms and functional factors $(N=188)$

\begin{tabular}{|c|c|c|c|}
\hline Symptoms (impairments) & Mean or $N$ & $\mathrm{SD}$ or $\%$ & Legend for models \\
\hline Pain & & & Pain \\
\hline Pain (BP-RAND-36: 0-100) & 67 & 27 & Randpain \\
\hline Muscle pain (VAS: 0-10) & 1.3 & 2.3 & Painvas \\
\hline Pain distribution (MRS: 0-100) & 7.2 & 12.6 & Painext \\
\hline Pain location (MRS: 0-45) & 2.9 & 5 & Painsite \\
\hline Pain worst (NRS: 0-10) & 2.3 & 8.2 & Painwors \\
\hline Psychological variables & & & Psycho \\
\hline Depression (HADS: 0-21) & 4 & 4.2 & Depress \\
\hline Anxiety (HADS: 0-21) & 5.3 & 4 & Anxiety \\
\hline Irritability (IQ: 4-16) & 7.6 & 2.7 & Irritab \\
\hline Mood (VAS: 0-10) & 8.3 & 2 & Moodvas \\
\hline Mental health (MHI-RAND-36: 0-100) & 68.7 & 19.3 & Mhealth \\
\hline Cognitive deficits (PDQ: 0-80) & 24.5 & 14.7 & $\operatorname{Cog}$ \\
\hline Decreased memory and attention (PASAT: 0-60) & 39.2 & 11.8 & Pasat \\
\hline \multicolumn{4}{|l|}{ Single-indicator components } \\
\hline Fatigue & 15.9 & 9.7 & Fatigue \\
\hline Neurological impairments (NI) & 6.3 & 2.3 & Neuro \\
\hline \multicolumn{4}{|l|}{ Functioning } \\
\hline Participation & & & Part \\
\hline Role physical (RP-RAND-36: 0-100) & 55.7 & 43.2 & Randphys \\
\hline Role emotional (RE-RAND-36: 0-100) & 67.7 & 40.2 & Randemo \\
\hline Social functioning (SF-RAND-36: 0-100) & 70.8 & 26 & Randsoc \\
\hline Social activities (PBMSI: 11-33) & 27.4 & 5.4 & Pbmsi \\
\hline Exercise barriers (EBS: $0-42$ ) & 20.3 & 15 & Barrier \\
\hline Illness intrusiveness (IIRS: 0-78) & 20.5 & 16.4 & Illint \\
\hline Work time & 25.3 & 18.3 & Paidwork \\
\hline Work activity & 51 & 28.8 & Carework \\
\hline Physical function & & & Function \\
\hline Physical functioning (PF-RAND-36: 0-100) & 67.8 & 31.3 & Randpfi \\
\hline Walking capacity (6MWT: meter) & 418 & 171 & Walktest \\
\hline Spasticity (MAS: 0-60) & 2.3 & 5.8 & Spastic \\
\hline Balance (EQUI: 0-20) & 17 & 4.9 & Balance \\
\hline Lower extremity power (jump height: $\mathrm{cm}$ ) & 62.7 & 39 & Jump \\
\hline Curl-ups $(\neq)$ & 84.3 & 87 & Curlup \\
\hline Push-ups $(\neq)$ & 26.8 & 41.5 & Pushup \\
\hline Grip strength (JTM D: kg) & 97.9 & 28 & Grip \\
\hline Gait speed (comfortable) $(\mathrm{m} / \mathrm{s})$ & 115.6 & 37.5 & Gaitcomf \\
\hline Gait speed (fast) (m/s) & 165.4 & 61.3 & Gaitfast \\
\hline Aerobic capacity (MCAFT: $\neq$ ) & 49.6 & 19.8 & Aerocap \\
\hline Upper extremity dysfunction (DASH: 0-84) & 16.3 & 19.3 & Dash \\
\hline
\end{tabular}

EBS Exercise Barriers Scale, IIRS Illness Intrusiveness Scale, PBMSI Preference-Based Multiple Sclerosis Index, $P F$ Physical Function Scale of RAND-36, $6 M W T$ six-minute walk test, MAS Modified Ashworth Scale, EQUI EQUI Balance Scale, JTMD Jamar TM Dynamometer, MCAFT Modified Canadian Aerobic Fitness Test, DASH Disabilities of the Arm, Shoulder and Hand, $P D Q$ Perceived Deficits Questionnaire, $P A S A T$ Paced Auditory Serial Addition Test, HADS Hospital Anxiety and Depression Scale, IQ Irritability Questionnaire, VAS Visual Analogue Scale, MHI-RAND-36 Mental Health Scale of RAND-36, BP-RAND36 Bodily Pain Subscale of RAND-36, MRS Margolis Rating System, NRS Numeric Rating Scale behavioral approach suggests that fatigue is worsened by the individual's interpretation of symptoms or associated symptoms and not only by disease severity $[55,56]$. The present study also showed that cognitive-behavioral factors are more involved in the persistence of MS fatigue than biological factors, such as neurological impairments or 


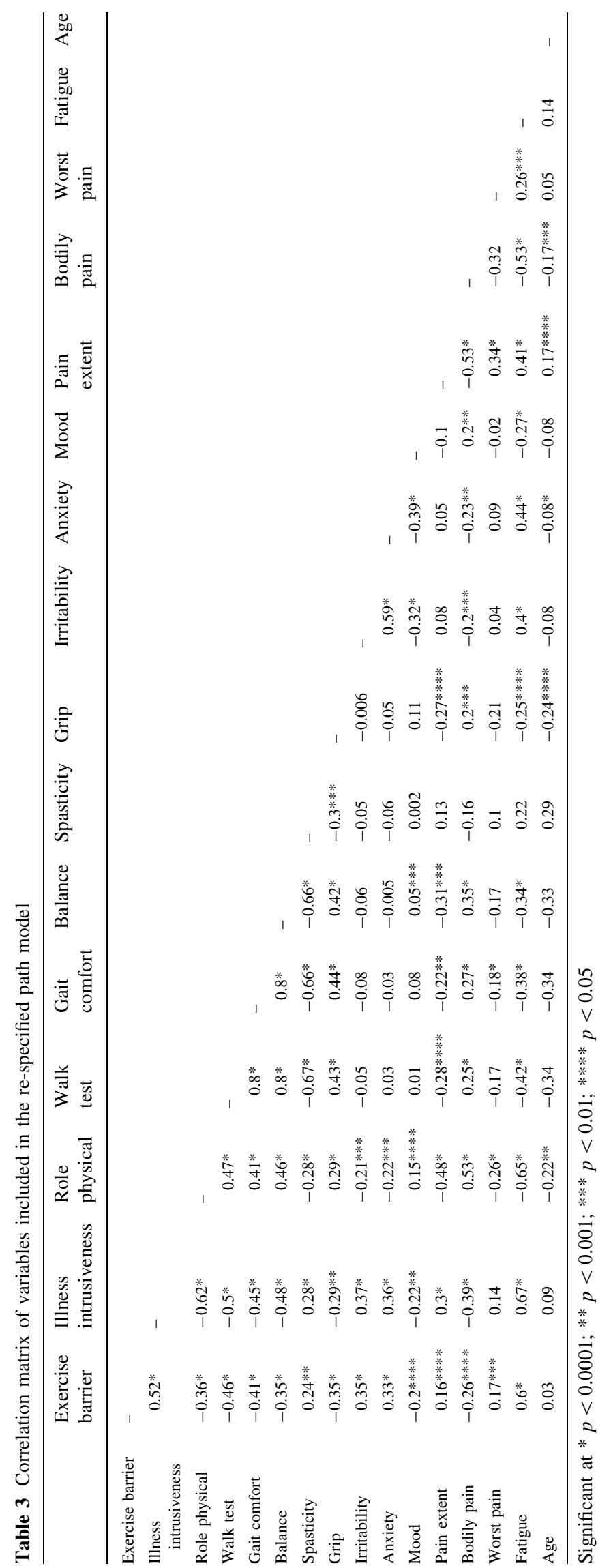


Table 4 Model progression

\begin{tabular}{|c|c|c|c|c|c|c|c|c|}
\hline Model & $\chi^{2}$ & $\begin{array}{l}\chi^{2} \\
p \text { value }\end{array}$ & SRMR & CFI & TLI & RMSEA & $\begin{array}{l}\text { RMSEA } \\
90 \% \text { CI }\end{array}$ & $\begin{array}{l}\text { RMSEA } \\
p \text { value }\end{array}$ \\
\hline Initial path model & 1532 & 0.00 & 0.1 & 0.77 & 0.75 & 0.1 & $0.09-0.10$ & 0.00 \\
\hline \multicolumn{9}{|l|}{$\begin{array}{l}32 \text { Variables representing the four latent variables (physical } \\
\text { function, psychological variables, pain, and participation), and } \\
\text { fatigue, NI, and age were included in the initial model (Fig. 1) }\end{array}$} \\
\hline Simplified path model & 419 & 0.000 & 0.08 & 0.89 & 0.87 & 0.08 & $0.07-0.09$ & 0.000 \\
\hline \multicolumn{9}{|l|}{$\begin{array}{l}\text { The RAND-PFI, DASH, gait speed (fast), jump test, MCAFT, } \\
\text { curl-ups, and push-ups were removed from the physical } \\
\text { function latent. Pain location and PASAT were removed from } \\
\text { the pain and the psychological variables components. RAND- } \\
\text { RE, PBMSI, work time, and work activity were removed from } \\
\text { the participation latent }\end{array}$} \\
\hline Re-specified path model & 147 & 0.005 & 0.05 & 0.97 & 0.96 & 0.7 & $0.07-0.09$ & 0.04 \\
\hline \multicolumn{9}{|l|}{$\begin{array}{l}\text { Direct effect of age on participation and fatigue as well as direct } \\
\text { effects of pain on physical function, psychological variables, } \\
\text { and participation were eliminated. Depression, RAND-MHI, } \\
\text { cognitive impairment, RAND-SF, and muscle pain were } \\
\text { removed from their related latent components }\end{array}$} \\
\hline Final model & 113.8 & 0.08 & 0.05 & 0.98 & 0.98 & 0.90 & $0.000-0.054$ & 0.03 \\
\hline $\begin{array}{l}\text { NI was dropped from the model. Correlations were added } \\
\text { between pain extension and pain worse intensity and between } \\
\text { RAND-RP and Barrier Scale (Fig. 2) }\end{array}$ & & & & & & & & \\
\hline
\end{tabular}

$\chi^{2}$ Chi-square test, $C I$ confidence interval, $N I$ neurological impairments

Fig. 2 Final model of participation

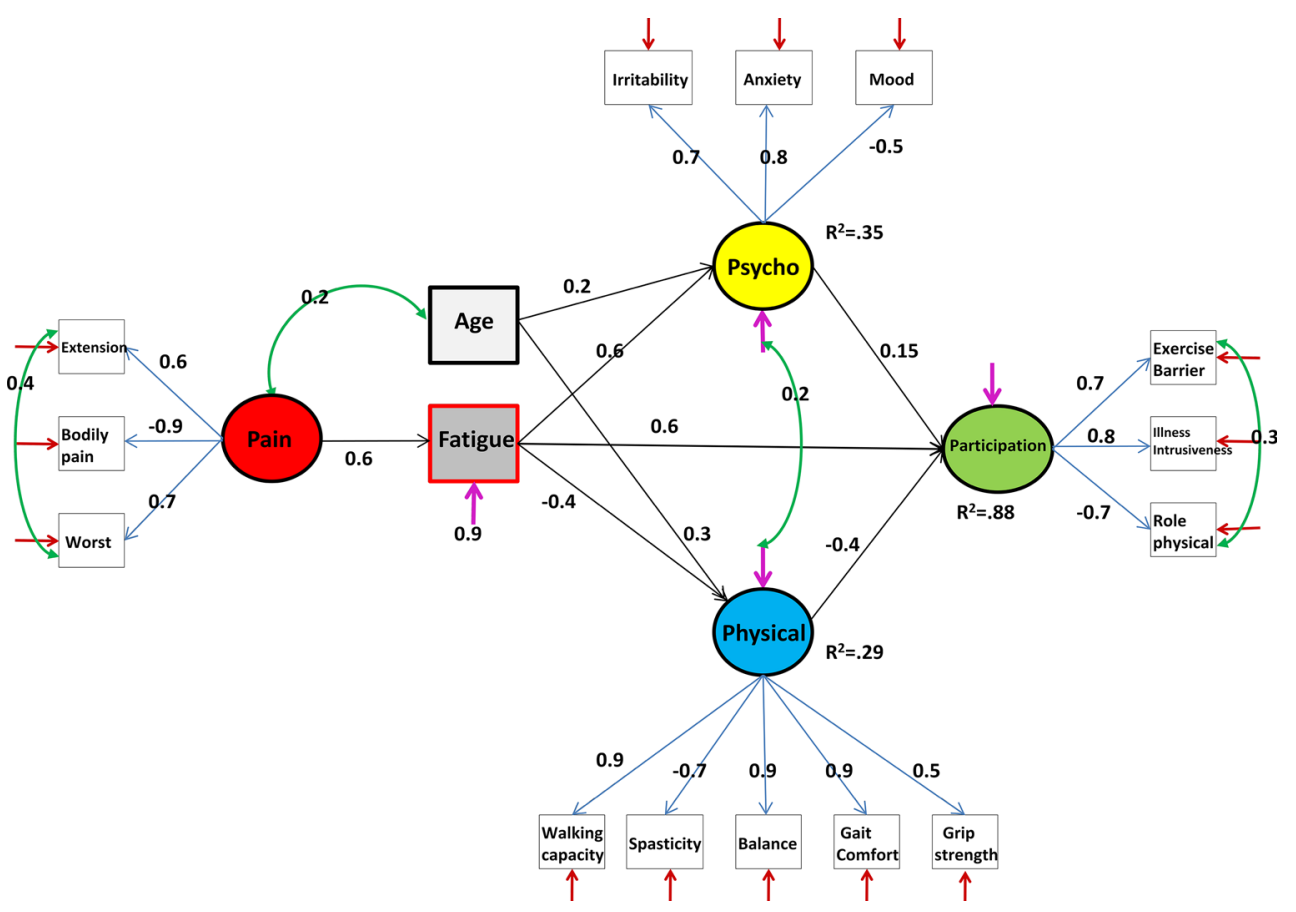

disease severity, as in our final model no relationship was found between those factors and fatigue.

In link with another study [12], the current study provided results for considering the role of physical function as an important correlate of social activities and participation in persons with MS. In persons with MS, difficulty with walking is related, either alone or in combination, to MS symptoms such as muscle weakness, spasticity, fatigue, pain, and depression [57]. These symptoms can produce problems with walking, unsteady gait, and difficulty with keeping balance and standing, thus avoiding participation in social activities. 
Table 5 Significant direct, indirect, and total effects of study variables on participation

Table 6 Maximum likelihood estimates of re-specified model

\begin{tabular}{llll}
\hline Study variables & Direct effect & Indirect effect & Total effect \\
\hline Physical function & $0.37^{*}$ & - & 0.37 \\
Psychological variables & $0.15^{* *}$ & - & 0.15 \\
Fatigue & $0.63^{*}$ & $0.14^{*}$ & 0.77 \\
Pain & - & $0.4^{*}$ (through fatigue) & 0.4 \\
Age & - & $0.12^{*}$ (through physical function) & 0.12 \\
\hline
\end{tabular}

Significant at $* p<0.0001$ or $* * p<0.05$

\begin{tabular}{|c|c|c|c|}
\hline & Estimate $(\beta)^{\mathrm{a}}$ & Standard error & Critical ratio $^{\mathrm{b}}$ \\
\hline \multicolumn{4}{|l|}{ Participation BY } \\
\hline Exercise barrier & 0.70 & 0.05 & 14.22 \\
\hline Illness intrusiveness & 0.79 & 0.04 & 21.75 \\
\hline Role physical & -0.75 & 0.05 & -16.46 \\
\hline \multicolumn{4}{|l|}{ Physical function BY } \\
\hline Walk test & 0.92 & 0.02 & 54.1 \\
\hline Gait comfortable & 0.88 & 0.03 & 34.5 \\
\hline Balance & 0.90 & 0.02 & 37.95 \\
\hline Spasticity & -0.73 & 0.05 & -14.52 \\
\hline Grip & 0.49 & 0.07 & 6.58 \\
\hline \multicolumn{4}{|l|}{ Psychological variables BY } \\
\hline Irritability & 0.72 & 0.07 & 9.67 \\
\hline Anxiety & 0.82 & 0.05 & 17.82 \\
\hline Mood & -0.46 & 0.07 & -6.35 \\
\hline \multicolumn{4}{|l|}{ Pain BY } \\
\hline Pain distribution & 0.62 & 0.06 & 9.89 \\
\hline Pain scale of RAND-36 & -0.86 & 0.05 & -17.1 \\
\hline Worst pain severity & 0.74 & 0.05 & 13.99 \\
\hline \multicolumn{4}{|l|}{ Participation ON } \\
\hline Physical function & -0.37 & 0.06 & -6.17 \\
\hline Psychological variables & 0.15 & 0.08 & 1.9 \\
\hline Fatigue & 0.63 & 0.07 & 8.5 \\
\hline \multicolumn{4}{|l|}{ Physical function ON } \\
\hline Fatigue & -0.38 & 0.06 & -6.39 \\
\hline Age & -0.33 & 0.07 & -4.63 \\
\hline \multicolumn{4}{|l|}{ Psychological variables ON } \\
\hline Fatigue & 0.59 & 0.06 & 9.31 \\
\hline Age & -0.18 & 0.07 & -2.43 \\
\hline \multicolumn{4}{|l|}{ Fatigue ON } \\
\hline Pain & 0.63 & 0.05 & 11.9 \\
\hline Physical function WITH psychological variables & 0.22 & 0.08 & 2.79 \\
\hline Pain WITH age & 0.21 & 0.07 & 2.9 \\
\hline Pain distribution WITH worst pain severity & 0.44 & 0.09 & 4.60 \\
\hline Exercise barrier WITH role physical & 0.34 & 0.09 & 3.47 \\
\hline
\end{tabular}

$B Y$ factor loading of indicator variables, $O N$ path coefficients, WITH associations

${ }^{\text {a }}$ Parameter estimates $(\beta)$ are interpreted in the same way as regression coefficients

b All critical ratios had significant results/paths
In contrast with another study [12], our result revealed that depression was not a predictor in the perpetuation of participation in people with MS. This can be partly related to the fact that our sample reported no serious depressive symptom (the mean scores of depression scores of our sample was 4 out of 21 on HADS). In addition, those 
studies used different measures to assess depression, such as the short form of the Center for Epidemiological Studies Depression (CES-D) Scale [12]. This brings up the concern of how different measures for assessing the same construct may find different results across different studies.

Results of this study further indicated the indirect effects of pain on participation. Pain is often considered as one of the MS symptoms that may contribute to problems with role participation [2, 14-18]. Thus, it is reasonable to think that in the presence of pain, people may be reluctant to engage in physical and social activities. The results of the current study, however, showed no direct effect of pain on participation. The only effect of pain on participation was through fatigue. The results of this study indicated that individuals with pain, who experienced higher levels of fatigue, reported lower levels of social participation. Fatigue and pain are related symptoms that often occur concurrently in people with MS and one can lead to or exacerbate the other [58]. These symptoms are possibly associated through common etiology due to the simultaneous damage to nerve fibers across different parts of the central nervous system (CNS) [59]. Pain could to some extent justify energy expenditure so as fear of pain could make someone experiencing them more tired. From a rehabilitation perspective, results from the current study provide useful information to help healthcare professionals, clinicians, and researchers to target symptoms that often occur together, so their concurrent appearance exacerbate their impact on disease consequences, e.g., role participation. Interventions that target fatigue through pain can also be designed to impact on other aspects of life such as role participation. For example, teaching of energy conservation techniques to alleviate pain during ambulation could reduce fatigue so as role participation.

Comparing our model with the WCM [20], we found that symptoms such as pain and fatigue were placed on the left side of the model, followed by the consequences of these symptoms on function, which then all together affect participation. What we found is also in agreement with Sullivan [29] and Mayo [60] who concluded from their SEM model in stroke that symptoms are important contributors to participation.

The current study has several strengths. The sample of study is a representative of the population living with MS in Canada due to the several reasons. First, it was randomly selected from three MS clinics in different areas of the Greater Montreal from populations who were culturally diverse including the whole range of disease severity and type, consistent with a clinical spectrum of MS [61]. Second, the sample consisted of substantially more women than men; the ratio of women to men in our study was
2.8:1, indicating that our sample is representative of gender difference in MS population (the ratio 3:1) [3]. Third, the mean age at which participants were diagnosed with MS was 43 years, which corresponds with the results reported by the MS Society of Canada [61]. Strength of this study further relates to the validity of the conceptualization of participation construct in MS population due to the full appropriate selection of participation measures, adequate research method, and complex statistical methods used.

This research had also some limitations. First, this was a cross-sectional study when subjects were assessed at one point in time. This limitation is particularly important in MS due to its progress course, as factors contributing to outcomes of interest change over time. Longitudinal studies are needed to evaluate changes in the participation over time and determine whether any changes are related to the factors identified in this study. Randomized controlled trials investigating the effects of different intervention approaches on participation and its contributors are also suggested. Second, as we only included persons diagnosed for MS since 1995, we acknowledge that these recruitment criteria may underrepresent symptoms and other functioning status in our sample. Since 1995, advances in neuroimaging techniques, such as MRI, and DMT facilitate earlier diagnosis of disease, and reduce the speed of disease progression [62]. Another limitation of this study relates to its relatively small sample size which is smaller than 200 cases as recommended in the literature [45]. However, as the current sample included 188 participants who were assumed to be a real representative of the MS population, due to the method of recruitment procedure and richness of data collected, we believe that the number of participants who included in this study was enough to provide a reliable estimate of parameters.

Future efforts need to assess the impact of more environmental factors, such as weather temperature, neighborhood, facilities, transportation, insurance, and health policy on participation. Expanding the assessment to include more MS-related symptoms and other personal factors such as societal attitudes, beliefs, satisfaction, self-efficacy, and coping strategy to the analysis is suggested as well. Research also needs to be done to go beyond role function and participation to health perception and QOL.

The results of this study verified how well the different measures captured different aspects of the multidimensional concept of participation construct, thus suggesting the need to have the multidisciplinary treatment approach for participation restriction. Identification of the strength of the contributors to participation would help prioritize intervention approaches for its improvement. For example, our results suggest that in persons with MS a careful assessment of 
fatigue, physical function, pain, and psychological variables would provide a much better guide to an individual's participation than any combination of biological and physiological variables or contextual factors. Although this conclusion will not surprise experienced clinicians, it does support the value of a thorough assessment and management of those specific types of symptoms. Unlike the pathological variables associated with MS, those symptoms may be modified to improve participation [52-54]. This information also is important for the planning of rehabilitation services, i.e., intervention approaches need to focus on reducing MS impairments rather than only shifting to a function-based approach, potentially increasing the opportunity for people with MS to optimize their HRQL. The role of fatigue in driving the path to participation indicates that its overall effect cannot be underestimated. Reducing fatigue may be the central treatment target. As mentioned previously, healthcare providers should consider a full spectrum of effective fatigue management approaches, from exercise to educational strategies and self-management techniques in conjunction with medication [53]. Given the impacts of pain on fatigue, there is a need for a thorough assessment and treatment of different dimensions of pain. Management of MS pain involves a variety of pharmacological and nonpharmacological approaches [63]. Massage, physical activity, yoga, tai chi, heat therapy, hydrotherapy, herbal remedies, acupuncture, and cognitive-behavioral therapy are examples of non-pharmacological approaches that have effectively been used in MS population to modify MS-related symptoms [2, 15-18, 50, 51, 59, 63].

The findings of this research play an important role in the recommendation to health policy makers to ensure that adequate supports are available for people with MS to maximize their participation at the life pursuits. Providing convenient facilities where people with MS can go to attend social and recreational activities, as well as the possibility of providing appropriate resources for patients, would increase their role participation. Giving appropriate information to patients about the advantage of participating in leisure, exercise, and social activities and guiding them to do these safely are also suggested.

\section{Conclusion}

Results of the current study suggested fatigue, physical function, psychological variables, pain, and age as the most important contributors to role participation of individuals with MS. An interesting result of this study was the central role that fatigue appeared to have on the social role of people living with MS. We indicated that despite advances in pharmacological and non-pharmacological treatment, still fatigue continues to be the most disabling symptom in persons with MS, thus highlighting the need for and importance of promoting interventions for the management of MS-related fatigue.

The optimum target of rehabilitation interventions is increasing participation in life situations, as it is known to be contributing strongly to QOL $[6,7]$. A clear understanding of participation and its predictors can ultimately improve QOL in individuals with MS. For example, if, as the final model of this study indicates, fatigue is reduced and participation is increased, then quality of life should also improve. This highlights that further research needs to be done to go beyond symptoms, role function, and participation to health perception and QOL.

Acknowledgments Shahrbanian was a recipient of the Canadian Institutes of Health Research Award (Grant No. 218665). Authors also acknowledge the contribution of Ayse Kuspinar, Shang Yuan Teng, and Elaine Roger, who recruited patients from MNH, CNRS, and CHUM, respectively.

\section{Compliance with ethical standards}

Conflict of interest The authors declare that there is no conflict of interest.

Ethical standard Study protocol and procedures were approved by the ethics committee of each participating hospital, and informed consent was obtained and signed by all subjects on the day of testing.

\section{Appendix}

See Table 7.

Table 7 Measures included in analyses

\begin{tabular}{llll}
\hline Variable & Construct $^{\mathrm{a}}$ & Measure & Scale \\
\hline Pain & & & \\
Pain severity & Symptom & Numeric Rating Scale (NRS) & Quasi-continuous \\
Muscle pain intensity & Symptom & Visual Analogue Scale (VAS) & Quasi-continuous \\
Bodily pain & Symptom & RAND-36 Health Survey Pain Subscale (BPS) & Continuous \\
Pain location & Symptom & Margolis rating system & Continuous \\
Pain distribution & Symptom & Margolis drawing rating system & Continuous \\
\hline
\end{tabular}


Table 7 continued

\begin{tabular}{|c|c|c|c|}
\hline Variable & Construct $^{\mathrm{a}}$ & Measure & Scale \\
\hline \multicolumn{4}{|l|}{ Psychological variables } \\
\hline Depression/anxiety & Symptom & Hospital Anxiety and Depression Scale (HADS) & Quasi-continuous \\
\hline Psychological health & Functional status & RAND-36 Health Survey Mental Health Index (MHI-5) & Continuous \\
\hline Mood & Symptom & Visual Analogue Scale (VAS) & Quasi-continuous \\
\hline Irritability & Symptom & Irritability questionnaire & Quasi-continuous \\
\hline Cognitive impairment & Symptom & Perceived Deficits Questionnaire (PDQ) & Continuous \\
\hline Processing speed/attention & Functional status & Paced auditory serial addition test (PASAT) & Continuous \\
\hline \multicolumn{4}{|l|}{ Single indicator } \\
\hline Fatigue & Symptom & RAND-36, MFIS, FAMS, MFI & Continuous \\
\hline Neurological impairments & Symptom & Symptom checklist, medical chart, EDSS & Continuous \\
\hline \multicolumn{4}{|l|}{ Participation } \\
\hline Role physical & Functional status & RAND-36 Role Physical Subscale (RP) & Continuous \\
\hline Role emotional & Functional status & RAND-36 Role Emotional Subscale (RE) & Continuous \\
\hline Social functioning & Functional status & RAND-36-Social Functioning Subscale (SF) & Continuous \\
\hline Work & Functional status & Socio-demographic questionnaire & Continuous \\
\hline Illness intrusiveness & Functional status & Illness Intrusiveness Rating Scale (IIRS) & Continuous \\
\hline Exercise barriers & Functional status & Exercise Barriers Scale (EBS) & Continuous \\
\hline Ability to accomplish work & Functional status & Preference-Based MS Index (PBMSI) & Continuous \\
\hline \multicolumn{4}{|l|}{ Physical function } \\
\hline Walking capacity & Functional status & Six-minute walk test & Continuous \\
\hline Walking speed & Functional status & Gait speed test & Continuous \\
\hline Lower extremity power & Functional status & Vertical jump test & Continuous \\
\hline Muscle stiffness and spasticity & Symptom & Modified Ashworth Scale (MAS) & Continuous \\
\hline Balance capacity & Symptom & EQUI Scale & Continuous \\
\hline Upper extremity dysfunction & Body structure and function & Disabilities of the Arm, Shoulder and Hand (DASH) & Continuous \\
\hline Grip strength & Functional status & Jamar TM dynamometer & Continuous \\
\hline Aerobic capacity & Functional status & The Modified Canadian Aerobic Fitness Test (MCAFT) & Continuous \\
\hline Upper limb muscle endurance & Functional status & The push-ups test & Continuous \\
\hline Abdominal muscle endurance & Functional status & Partial curl-ups test & Continuous \\
\hline Physical functioning & Functional status & RAND-36 Physical Functioning Subscale (PFI) & Continuous \\
\hline
\end{tabular}

${ }^{a}$ Construct of each variable has been chosen based on the components of Wilson-Clearly model [20]

\section{References}

1. Noseworthy, J. H., Lucchinetti, C., Rodriguez, M., \& Weinshenker, B. G. (2000). Multiple sclerosis. New England Journal of Medicine, 343, 938-952.

2. O'Connor, P. (2009). Key issues in the diagnosis and treatment of multiple sclerosis: An overview. Neurology, 59, S1-S33.

3. Richards, R. G., Sampson, F. C., Beard, S. M., \& Tappenden, P. (2002). A review of the natural history and epidemiology of multiple sclerosis: Implications for resource allocation and health economic models. Health Technology Assessment, 6(10), 1-73.

4. Devins, G. M., Dion, R., Pelletier, L. G., Shapiro, C. M., Abbey, S., \& Raiz, L. R. (2001). Structure of lifestyle disruptions in chronic disease, a confirmatory factor analysis of the Illness Intrusiveness Ratings Scale. Medical Care, 39(10), 1097-1104.

5. Neubeck, L., Freedman, S. B., Clark, A. M., Briffa, T., Bauman, A., \& Redfern, J. (2012). Participating in cardiac rehabilitation, a systematic review and meta-synthesis of qualitative data. European Journal of Preventive Cardiology, 19(3), 494-503.
6. Barclay-Goddard, R., Ripat, J., \& Mayo, N. (2012). Developing a model of participation post-stroke, a mixed-methods approach. Quality of Life Research, 21, 417-426.

7. Mayo, N. E., Wood-Dauphinee, S., Cote, R., Durcan, L., \& Carlton, J. (2002). Activity, participation, and quality of life six months post-stroke. Archives of Physical Medicine and Rehabilitation, 83, 1035-1042.

8. World Health Organization. (2001). International classification of functioning, disability and health (2nd revision ed.). http:// www3.who.int/icf/icftemplate.cfm. Geneva.

9. Salter, K., Jutai, J. W., Teasell, R., Foley, N. C., Bitensky, J., \& Bayley, M. (2005). Issues for selection of outcome measures in stroke rehabilitation, ICF participation. Disability and Rehabilitation, 27(9), 507-528.

10. Johnston, M. V., \& Miklos, C. S. (2002). Activity-related quality of life in rehabilitation and traumatic brain injury. Archives of Physical Medicine and Rehabilitation, 83(Suppl 2), S26-S38.

11. Whiteneck, G. G., Charliefue, S. W., \& Gerhart, K. A. (1992). Quantifying handicap, a new measure of long-term rehabilitation 
outcomes. Archives of Physical Medicine and Rehabilitation, 73, 519-526.

12. Phillips, L., \& Stuifbergen, A. (2009). Structural equation modeling of disability in women with fibromyalgia or multiple sclerosis. Western Journal of Nursing Research, 31, 89-109.

13. Yorkston, K., Johnson, K., \& Klasner, E. (2005). Taking part in life, enhancing participation in multiple sclerosis. Physical Medicine \& Rehabilitation Clinics of North America, 16, 583-594.

14. Shahrbanian, S., Auais, M., Duquette, P., Andersen, K., \& Mayo, N. E. (2013). Does pain in individuals with multiple sclerosis affect employment? A systematic review and meta-analysis. Pain Research \& Management, 18(5), e94-e100.

15. Ehde, D. M., Osborne, T. L., \& Jensen, M. P. (2005). Chronic pain in persons with multiple sclerosis. Physical Medicine \& Rehabilitation Clinics of North America, 16, 503-512.

16. Archibald, C. J., McGrath, P. J., \& Ritvo, P. G. (1994). Pain prevalence, severity and impact in a clinic sample of multiple sclerosis patients. Pain, 58, 89-93.

17. Hadjimichael, O., Kerns, R. D., Rizzo, M. A., Cutter, G., \& Vollmer, T. (2007). Persistent pain and uncomfortable sensations in persons with multiple sclerosis. Pain, 127, 35-41.

18. Warnell, P. (1991). The pain experience of a multiple sclerosis population, a descriptive study. Axon, 13, 26-28.

19. Kerns, R., Kassirer, M., \& Otis, J. (2002). Pain in multiple sclerosis, a biopsychosocial perspective. Journal of Rehabilitation Research and Development, 39, 225-232.

20. Wilson, I. B., \& Cleary, P. D. (1995). Linking clinical variables with health-related quality of life. A conceptual model of patient outcomes. JAMA, 273(1), 59-65.

21. Kurtzke, J. F. (1983). Rating neurologic impairment in multiple sclerosis, an expanded disability status scale (EDSS). Neurology, 33(11), 1444-1452.

22. Ng, P., Finch, L., Hum, S., \& Mayo, N. E. (2013). The path to work capacity in people with multiple sclerosis. Dissertation, McGill University.

23. Jensen, M. P., Turner, J. A., \& Romano, J. M. (1999). Comparative reliability and validity of chronic pain intensity measures. Pain, 83, 157-162.

24. Margolis, R. B., Tait, R. C., \& Krause, S. J. (1986). A rating system for use with patient pain drawings. Pain, 24, 57-65.

25. Hays, R. D., Sherbourne, C. D., \& Mazel, R. M. (1993). The RAND 36-item health survey 1.0. Health Economics, 2(3), 217-227.

26. Ware, J. E, Jr. (1987). Standards for validating health measures: Definition and content. Journal of Chronic Diseases, 40(6), 473-480.

27. Bjelland, I., Dahl, A. A., Haug, T. T., \& Neckelmann, D. (2002). The validity of the hospital anxiety and depression scale, an updated literature review. Journal of Psychosomatic Research, 52, 69-77.

28. Illfeld, F. W. (1976). Further validation of a psychiatric symptom index in a normal population. Psychological Reports, 39, $1215-1228$

29. Sullivan, J. L., Edgley, K., \& Dehoux, E. (1990). A survey of multiple sclerosis. Part 1: Perceived cognitive problems and compensatory strategy use. Canadian Journal of Rehabilitation, 4(2), 99-105.

30. Gronwall, D. M. (1977). Paced auditory serial-addition task, a measure of recovery from concussion. Perceptual and Motor Skills, 44(2), 367-373.

31. Cella, D. F., Dineen, K., Arnason, B., Reder, A., Webster, K. A., \& Karabatsos, G. (1996). Validation of the functional assessment of multiple sclerosis quality of life instrument. Neurology, 47, 129-139.

32. Ritvo, P., Fischer, J. S., Miller, D., Andrews, H., Paty, D., \& LaRocca, N. G. (1997). Multiple sclerosis quality of life, A user's manual. Denver: National Multiple Sclerosis Society.
33. Weinshenker, B. G., Bass, B., Rice, G. P. A., Noseworthy, J., Carriere, W., Baskerville, J., et al. (1989). The natural history of multiple sclerosis: A geographically based study. Brain, 112, 133-146.

34. Becker, H., Stuifbergen, A. K., \& Sands, D. (1991). Development of a scale to measure barriers to health promotion activities among persons with disabilities. American Journal of Health Promotion, 5(6), 44-454.

35. Poissant, L., Mayo, N. E., Wood-Dauphinee, S., \& Clarke, A. E. (2003). The development and preliminary validation of a preference-based stroke index (PBSI). Health and Quality of Life Outcomes, 1, 15-43.

36. Devins, G. M. (2010). Using the illness intrusiveness ratings scale to understand health-related quality of life in chronic disease. Journal of Psychosomatic Research, 68(6), 591-602.

37. Goldman, M. D., Marrie, R. A., \& Cohen, J. A. (2008). Evaluation of the six-minute walk in multiple sclerosis subjects and healthy controls. Multiple Sclerosis Journal, 14(3), 383-390.

38. Tyson, S., \& Connell, L. (2009). The psychometric properties and clinical utility of measures of walking and mobility in neurological conditions, a systematic review. Clinical Rehabilitation, 23(11), 1018-1033.

39. Tesio, L., Perucca, L., Franchignoni, F. P., \& Battaglia, M. A. (1997). A short measure of balance in multiple sclerosis, validation through Rasch analysis. Functional Neurology, 12(5), 255-265.

40. Bohannon, R. W., \& Smith, M. B. (1987). Interrater reliability of a modified Ashworth scale of muscle spasticity. Physical Therapy, 67(2), 206-207.

41. Health, C. S. E. P., Appraisal, F. P. H.-R., \& Strategy, C. (2004). The Canadian physical activity, fitness and lifestyle approach (3rd ed.). Ontario: Canadian Society for Exercise Physiology.

42. Markovic, G., Dizdar, D., Jukic, I., \& Cardinale, M. (2004). Reliability and factorial validity of squat and countermovement jump tests. Journal of Strength and Conditioning Research, 18(3), 551-555.

43. Mathiowetz, V., Weber, K., Volland, G., \& Kashman, N. (1984). Reliability and validity of grip and pinch strength evaluations. Journal of Hand Surgery. American Volume, 9(2), 222-226.

44. Cano, S., Barrett, L., Zajicek, J., \& Hobart, J. (2011). Beyond the reach of traditional analyses, using Rasch to evaluate the dash in people with multiple sclerosis. Multiple Sclerosis Journal, 17, 214-222.

45. Kline, R. B. (2011). Principles and practice of structural equation modeling (3rd ed.). New York: Guilford.

46. Hu, L., \& Bentler, P. M. (1999). Cutoff criteria for fit indexes in covariance structure analysis, conventional criteria versus new alternatives. Structural Equation Modeling, 6, 1-55.

47. MacCallum, R. C., Browne, M. W., \& Sugawara, H. M. (1996). Power analysis and determination of sample size for covariance structure modeling. Psychological Methods, 1, 130-149.

48. Muthén, L. K., \& Muthén, B. O. (1998-2012). Mplus User's Guide (7th ed.). Los Angeles, CA: Muthén \& Muthén.

49. SAS 9.2 (computer program). (2008). Version 9.2. Cary, NC, SAS Institute Inc.

50. Kempen, J. C. E., de Groot, V., Knol, D. L., Lankhorst, G. J., \& Beckerman, H. (2012). Self-reported fatigue and energy cost during walking are not related in patients with multiple sclerosis. Archives of Physical Medicine and Rehabilitation, 93(5), 889-895.

51. Khan, F., Amatya, B., \& Galea, M. (2014). Management of fatigue in persons with multiple sclerosis. Frontiers in Neurology, 15(5), 177

52. Lapierre, Y., \& Hum, S. (2007). Treating fatigue. The International MS Journal, 14, 64-71.

53. Asano, M., \& Finlayson, M. L. (2014). Meta-analysis of three different types of fatigue management interventions for people with multiple sclerosis: Exercise, education, and medication. 
Multiple Sclerosis International,. doi:10.1155/2014/798285. (Epub 2014 May 14).

54. Asano, M., Berg, E., Johnson, K., Turpin, M., \& Finlayson, M. L. (2014). A scoping review of rehabilitation interventions that reduce fatigue among adults with multiple sclerosis. Disability and Rehabilitation, 37(9), 729-738.

55. Skerrett, T. N., \& Moss-Morris, R. (2006). Fatigue and social impairment in multiple sclerosis, the role of patients' cognitive and behavioral responses to their symptoms. Journal of Psychosomatic Research, 61(5), 587-593.

56. Van Kessel, K., \& Moss-Morris, R. (2006). Understanding multiple sclerosis fatigue, a synthesis of biological and psychological factors. Journal of Psychosomatic Research, 61(5), 583-585.

57. Motl, R. W., Snook, E. M., \& Schapiro, R. T. (2008). Symptoms and physical activity behavior in individuals with multiple sclerosis. Research in Nursing \& Health, 31, 466-475.

58. Shahrbanian, S., Duquette, P., Kuspinar, A., \& Mayo, N. E. (2015). Consequences of symptom cluster in individuals with multiple sclerosis. Quality of Life Research Journal, 24(3), 617-629.
59. Lublin, F. D., \& Reingold, S. C. (1996). Defining the clinical course of multiple sclerosis: results of an international survey. National multiple sclerosis society (USA) advisory committee on clinical trials of new agents in multiple sclerosis. Neurology, 46(4), 907-911.

60. Mayo, N. E., Scott, S. C., \& Bayley, M. (2015). Modeling healthrelated quality of life in people recovering from stroke. Quality of Life Research, 24(1), 41-53.

61. Multiple Sclerosis Society of Canada. (2009). www.mssociety.ca. Canada, MS Society of Canada.

62. Mayo, N. (2008). Setting the agenda for multiple sclerosis rehabilitation research. Multiple Sclerosis Journal, 14(9), $1154-1156$.

63. Leary, S., \& Thompson, A. (2000). Current management of multiple sclerosis. International Journal of Clinical Practice, 54, $161-169$. 